\title{
Bandwidth-Adaptive Waveforms for Dynamic Spectrum Access Networks
}

\author{
Sutton P.D., Ozgul B., Nolan K.E., Doyle L.E. \\ Centre for Telecommunications Value-Chain Research (CTVR) \\ University of Dublin, Trinity College, Ireland \\ Email: suttonpd@tcd.ie, baris.ozgul@ctvr.ie, keith.nolan@ctvr.ie, ledoyle@tcd.ie
}

\begin{abstract}
This paper presents a novel solution to the challenge of waveform bandwidth estimation in DySPAN systems using intentionally embedded cyclostationary signatures.

Dynamic spectrum access networks (DySPANs) achieve high levels of spectrum use efficiency by exploiting spectrum white spaces, radio frequencies which are unused at given times and places. These white spaces have bandwidths which may not be known in advance and so, in order to fully exploit them, a DySPAN system must employ bandwidth-adaptive waveforms. It has been shown that multi-carrier transmission schemes can be leveraged to generate waveforms of flexible bandwidth. However, robust bandwidth estimation techniques are required for receiving nodes to successfully detect these signals and establish communication links.

This paper makes three key contributions. Firstly, a novel technique for the generation of bandwidth-adaptive multicarrier waveforms is proposed. Secondly, two approaches for parametrization of these bandwidth-adaptive waveforms in receiving DySPAN nodes are presented. The advantages and disadvantages of both bandwidth estimation techniques are examined and comparisons are drawn with approaches adopted for the IEEE 802.16 and draft IEEE 802.22 standards. Finally, the performance of both techniques under multipath fading conditions are examined using simulation results.
\end{abstract}

\section{INTRODUCTION}

Legacy approaches to spectrum management and inefficient spectrum use has given rise to the apparent spectrum scarcity currently experienced by regulators around the world. This apparent scarcity has resulted in high spectrum license costs, entry barriers for new wireless systems and services and stunted wireless innovation.

Enabled by emerging software-defined radio and cognitive radio technologies, Dynamic Spectrum Access Networks (DySPANs), offer a solution to the issue of inefficient spectrum use and apparent spectrum scarcity. DySPANs achieve new levels of spectrum use efficiency by exploiting spectrum white spaces, radio frequencies which are temporarily unused at given times and places.

One of the key challenges posed by the use of white space spectrum in this way is the need for highly flexible waveforms. Upon detection of a spectrum opportunity or white space, a DySPAN system must be capable of tailoring the carrier frequency and occupied bandwidth of its waveform to exploit that white space efficiently. Here the occupied bandwidth (OBW) is defined to be the bandwidth containing $99 \%$ of the total integrated power of the transmitted spectrum, centered on the carrier frequency. Proposed approaches for achieving this type of flexibility include the use of multi-carrier waveforms, particularly OFDM [1] and, more recently, filterbank-based waveforms [2] and multi-carrier CDMA [3]. Using multicarrier waveforms, flexible bandwidth signals can be achieved through activation and deactivation of individual subcarriers. In this way, the waveform can be sculpted to suit the white space spectrum [4].

Transmitting DySPAN nodes can generate highly flexible waveforms using adaptive multi-carrier schemes. However, in order for a node to receive that waveform and establish a communication link, robust techniques are required to detect and estimate the parameters of these waveforms. While static control channels could be employed to achieve this, they typically require dedicated frequency assignments and form a single point of failure for the network.

This paper shows that the establishment and maintenance of communication links using bandwidth-adaptive waveforms can be achieved using intentionally embedded cyclostationary signatures. Cyclostationary signatures are features which may be intentionally embedded in the physical properties of transmitted multi-carrier signals. They avoid the need for statically assigned control channels, may be generated using low-complexity transmitter architectures and provide a robust tool for signal detection, identification and frequency acquisition [5], [6].

The use of cyclostationary signatures to support bandwidthadaptivity involves multi-carrier waveforms which are generated using one or more bandwidth units (BUs), each consisting of a fixed number of subcarriers and containing an embedded cyclostationary feature. By detecting these embedded features, receiving nodes can determine the occupied bandwidth of the transmitted waveform and thus establish a communication link. Two approaches for using embedded features in this way are proposed. Using the first approach, receiving nodes detect each feature embedded in a given waveform and calculate the signal bandwidth using this information. In contrast, the second approach exploits the subchannelization which occurs as a result of the use of BUs. Each of these subchannels can be used to transmit a short control header containing information on the current carrier frequency and OBW being used by the overall signal. By detecting a single embedded feature, receiving nodes can synchronize with one of these subchannels, extract the control header and determine the properties of the overall waveform. This paper compares the 
advantages and disadvantages of both approaches and presents simulation results illustrating the performance of both under conditions of multipath fading.

The paper is organized as follows. Section II begins with a short review of cyclostationary signal analysis. A lowcomplexity technique for intentionally embedding cyclostationary features or signatures in multi-carrier signals is introduced and a suitable detector design is proposed. Section III discusses the use of bandwidth units (BUs) and embedded cyclostationary features to realize bandwidth-adaptive multicarrier waveforms for dynamic spectrum access. Two approaches for exploiting these features in receiving nodes are presented and compared. Section IV describes Monte Carlo simulations carried out to examine the performance of both approaches under multipath fading conditions. Results of these simulations are presented and used to further compare both bandwidth estimation approaches. Finally, Section V concludes.

\section{Cyclostationary Signatures}

\section{A. Cyclostationary Signal Analysis}

A signal $x(t)$ is defined to be second order cyclostationary (in the wide sense) if its autocorrelation function,

$$
R_{x}(t, \tau)=E\{x(t+\tau / 2) x(t-\tau / 2)\}
$$

is periodic in time $t$ for each time lag $\tau$. These periodicities are examined using the cyclic autocorrelation function (CAF) [7],

$$
\begin{aligned}
R_{x}^{\alpha}(\tau)= & \lim _{\Delta t \rightarrow \infty} \frac{1}{\Delta t} \int_{-\Delta t / 2}^{\Delta t / 2} x(t+\tau / 2) \\
& \cdot x(t-\tau / 2) e^{-i 2 \pi \alpha t} d t
\end{aligned}
$$

for cyclic frequency $\alpha$ and measurement interval $\Delta t$.

Second order cyclostationarity gives rise to specific correlation patterns which occur in the spectrum of the signal. These patterns may be used equivalently to examine the cyclostationarity of the signal and may be analyzed using the spectral correlation function (SCF) [7],

$$
\begin{aligned}
S_{x}^{\alpha}(f)=\lim _{\Delta f \rightarrow \infty \Delta t \rightarrow \infty} \lim _{\Delta t} \frac{1}{\Delta t} \int_{-\Delta t / 2}^{\Delta t / 2} \Delta f X_{1 / \Delta f}\left(t, f+\frac{\alpha}{2}\right) \\
\cdot X_{1 / \Delta f}^{*}\left(t, f-\frac{\alpha}{2}\right) d t
\end{aligned}
$$

where

$$
X_{1 / \Delta f}(t, v)=\int_{t-1 / 2 \Delta f}^{t+1 / 2 \Delta f} x(u) e^{-i 2 \pi v u} d u
$$

represents the complex envelope of the narrow-band-pass component of $x(t)$ with centre frequency $v$ and bandwidth $\Delta f$.

The spectral coherence (SC) [8], $C_{x}^{\alpha}$, is used to normalize the cyclic spectrum estimates in the range $[0,1]$ :

$$
C_{x}^{\alpha}(f)=\frac{S_{x}^{\alpha}(f)}{\sqrt{S_{x}^{0}(f+\alpha / 2) S_{x}^{0}(f-\alpha / 2)}}
$$

where $S_{x}^{0}(f)$ is the SCF at cyclic frequency $\alpha=0$.

\section{B. Signature Generation And Detection For OFDM}

Cyclostationary features may arise due to the inherent processes used in the generation of a telecommunications signal, however it has been shown that features may also be intentionally embedded in signals in order to facilitate signal detection and network identification and to aid frequency acquisition [6]. These intentionally embedded features are termed cyclostationary signatures.

Second order cyclostationarity manifests itself as a distinctive correlation pattern in the spectrum of the signal of interest. Therefore, in order to artificially embed a cyclostationary feature in a signal, one approach is to intentionally generate such a correlation pattern.

Orthogonal Frequency Division Multiplexing (OFDM) provides a highly spectrum efficient and robust transmission scheme due to the use of closely spaced, orthogonal carriers. It has been shown that the spectrum of an OFDM signal may be effectively sculpted by manipulating the data symbols transmitted upon these carriers [1] [9]. The unique ability to sculpt the spectrum of an OFDM signal in this way permits correlation patterns to be artificially created and thus artificial cyclostationary features or cyclostationary signatures to be embedded in a transmitted signal.

OFDM signals may be represented as a composite of $J$ statistically independent subchannel QAM signals [10]:

$$
w(t)=\sum_{\ell} \sum_{j=0}^{J-1} \gamma_{j, \ell} e^{i\left(2 \pi / T_{s}\right) j t} q(t-\ell T)
$$

where $w(t)$ is the complex envelope of an OFDM signal with a cyclic prefix, $\gamma_{j, \ell}$ is the IID message symbol transmitted upon subcarrier $j$ during OFDM symbol $\ell, J$ is the total number of subcarriers and $q(t)$ is a square shaping pulse of duration $T . T_{s}$ is the source symbol length and $T_{g}$ is the cyclic prefix length such that $T=T_{s}+T_{g}$.

Due to the statistical independence of the subchannel QAM signals, the problem of cyclostationary analysis of OFDM may be reduced to the analysis of these QAM signals. In the absence of a cyclic prefix, subcarrier orthogonality causes destruction of the individual QAM signal cyclostationarity. However, the use of a cyclic prefix causes a loss of subcarrier orthogonality and permits inherent QAM signal features to be detected [10].

Thus, the cyclic prefix typically used in OFDM waveforms gives rise to inherent cyclostationary features. However, in order to generate an artificial cyclostationary signature, a distinctive correlation pattern must be created in the spectrum of the signal. OFDM spectrum sculpting permits such a correlation pattern to be intentionally created by manipulating the message symbols $\gamma_{j, \ell}$, assigned to individual subcarriers. By mapping a set of subcarriers onto a second set as:

$$
\gamma_{j, \ell}=\gamma_{j+p, \ell}, \quad n \in \mathcal{M}
$$

where $\mathcal{M}$ is the set of subcarrier values to be mapped with cardinality $M$ and $p$ is the number of subcarriers between 


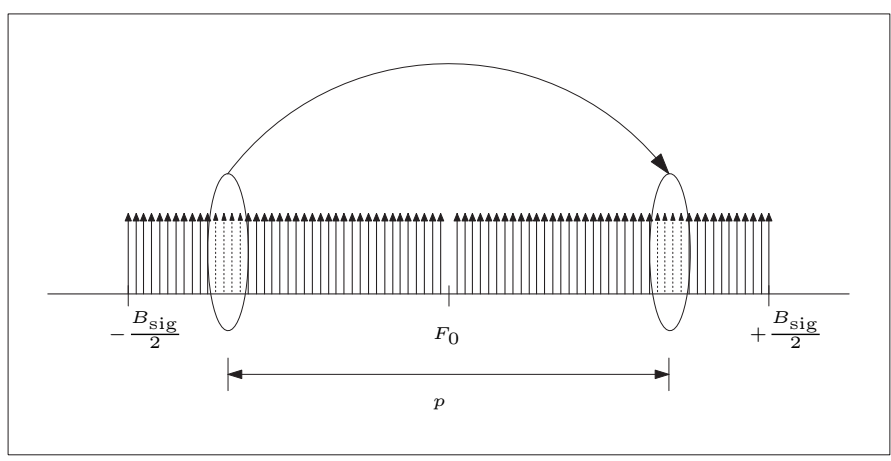

Fig. 1. Generation of a Cyclostationary Signature using OFDM Subcarrier Set Mapping.

mapped symbols, message symbols are redundantly transmitted on more than one subcarrier, a correlation pattern is created and a cyclostationary feature is embedded in the signal. Fig. 1 illustrates this process of subcarrier set mapping.

For an OFDM signal with a cyclic prefix, containing an intentionally embedded cyclostationary signature, the spectral correlation can be derived as [6]:

$$
S_{w}^{\alpha}(f)=\left\{\begin{array}{c}
\frac{\delta_{\gamma}^{2}}{T} \sum_{j=0}^{J-1} Q\left(f-\frac{j}{T_{s}}+\frac{\alpha}{2}\right) \cdot Q^{*}\left(f-\frac{j}{T_{s}}-\frac{\alpha}{2}\right), \\
\alpha=\frac{\ell}{T} \\
\frac{\delta_{\gamma}^{2}}{T} \sum_{j=0}^{J-1} Q\left(f-\frac{j}{T_{s}}+\frac{\alpha}{2}\right) \cdot Q^{*}\left(f-\frac{j+p}{T_{s}}-\frac{\alpha}{2}\right), \\
\alpha=\frac{p}{T_{s}} \pm \frac{\ell}{T}, j \in \mathcal{M} \\
\alpha \neq \frac{\ell}{T}, \frac{p}{T_{s}} \pm \frac{\ell}{T}
\end{array}\right.
$$

where $\mathcal{M}$ is the set of mapped subcarriers and where

$$
Q(f)=\frac{\sin (\pi f T)}{\pi f}
$$

is the Fourier transform of the square shaping pulse $q(t)$. The SCF for an OFDM signal containing an embedded cyclostationary signature is illustrated in Fig. 2 where $p=6, J=16$, $T_{g}=\frac{1}{4} T_{s}$ and a single subcarrier is mapped. The strong feature which is associated with the cyclostationary signature can be seen at cyclic frequency $\alpha=\frac{p}{T_{s}}=\frac{7.5}{T}$.

In order for embedded cyclostationary signatures to be of practical use, receiving nodes must be capable of detecting and analyzing them. Gardner showed that optimum cyclostationary feature detection is performed through correlation of the estimated SCF with the ideal SCF [7]:

$$
y_{\alpha}(t)=\int_{-\infty}^{\infty} S_{s}^{\alpha}(f) * \tilde{S}_{x}^{\alpha}(f) d f e^{i 2 \alpha \pi t}
$$

where $\tilde{S}_{x}^{\alpha}(f)$ is the estimated SCF following notch filtering to remove strong narrow-band interference.

Estimation of the SCF may be performed using the timesmoothed cyclic cross periodogram (TS-CCP), a consistent,

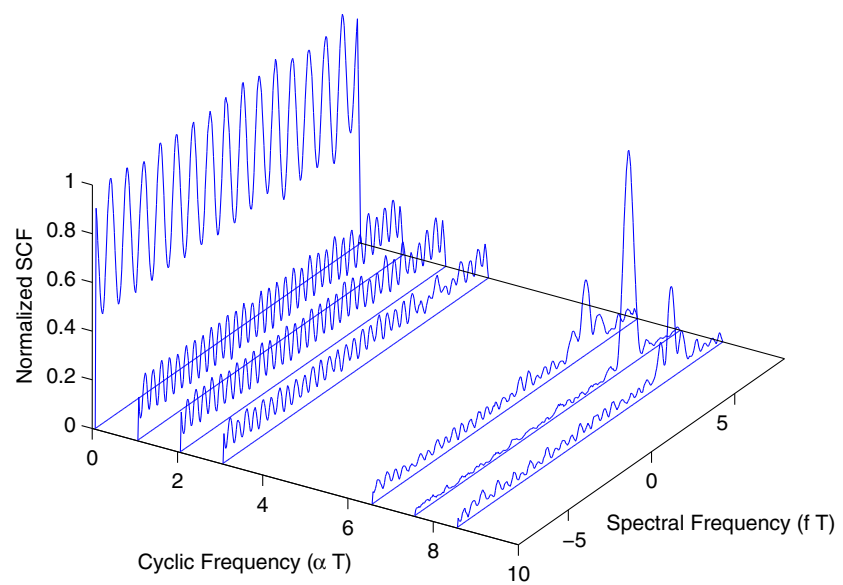

Fig. 2. Normalized SCF for OFDM with cyclic prefix and embedded cyclostationary signature.

asymptotically unbiased and complex normally distributed estimator for the cyclic cross spectrum [11]:

$$
\hat{S}_{x}^{\alpha}[k]=\frac{1}{L} \sum_{l=0}^{L-1} X_{l}[k] X_{l}^{*}[k-\alpha] W[k]
$$

where $W[k]$ denotes a smoothing spectral window and $X_{l}[k]$ is the Fourier transform of the discrete-time signal $x[n]$ after sampling the received signal $x(t)$,

$$
X_{l}[k]=\sum_{n=0}^{N-1} x[n] \exp \frac{-i 2 \pi n k}{N}
$$

Estimates are calculated using $L$ windows of length $N$ where $N$ is the duration of a single OFDM symbol.

Cyclostationary features generated through OFDM subcarrier set mapping may be successfully detected using spectral resolution $\Delta f$, equal to the OFDM subcarrier spacing. Using this approach, the ideal spectral correlation function may be approximated using a simple rectangular window of width $M . \Delta f$, where $M$ is the number of subcarriers in the mapped set $\mathcal{M}$. In this way, a low-complexity single-cycle signature detector may be implemented as:

$$
y_{\alpha}=\max _{0 \leq k \leq N-1} \sum_{m=0}^{M-1} H[m] \hat{C}_{x}^{\alpha}[k-m]
$$

where $H[m]$ is a rectangular window and $\hat{C}_{x}^{\alpha}[k]$ is computed by modifying (5) as

$$
\hat{C}_{x}^{\alpha}[k]=\frac{\hat{S}_{x}^{\alpha}[k]}{\sqrt{\hat{S}_{x}^{0}[k] \hat{S}_{x}^{0}\left[(k-\alpha)_{N}\right]}}
$$

with $(.)_{N}$ denoting the modulo- $N$ operation. Then, $y_{\alpha}$ is compared to a threshold for feature detection. 


\section{BANDWIDTH ADAPTIVE WAVEFORMS}

The key challenge associated with the use of bandwidth adaptive waveforms for DySPAN systems is the estimation of that occupied bandwidth at receiving nodes. It has been shown that cyclostationary signatures can provide a highly flexible tool for physical layer signalling in multi-carrier systems and can be detected and analyzed by receivers with minimum information about the signal of interest and without the need for time or frequency synchronization. These signatures can be leveraged to achieve signal detection, identification and frequency acquisition. When employed in bandwidth adaptive waveforms, these signatures can also be used to perform robust OBW estimation.

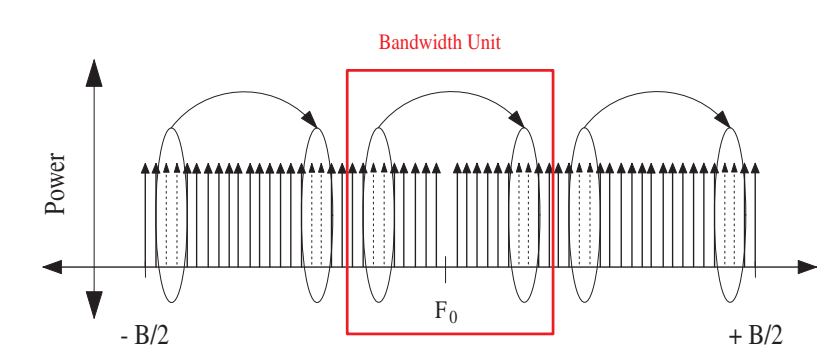

Fig. 3. Generation of a signal using multiple bandwidth units (BUs), each containing an independent cyclostationary signature.

The proposed approach for using cyclostationary signatures in bandwidth adaptive multi-carrier waveforms is illustrated in Fig. 3. Transmitted waveforms are generated using an integer multiple of bandwidth units (BUs), each containing a fixed number of subcarriers at fixed spacings. Within each of these units, an independent cyclostationary signature is embedded. Each signature is generated using the same distance between mapped subcarrier sets $p$ (see eq. 7). In this way, a waveform comprising more than one unit will contain more than one cyclostationary feature, each at a different spectral frequency, $f$, but the same cyclic frequency, $\alpha$. Fig. 4 illustrates the normalized SCF for an OFDM waveform generated in this way using three BUs and where $p=8, J=16$ for each unit. In this example, a single subcarrier is mapped and a $\frac{1}{8}$ cyclic prefix is used.

\section{A. Bandwidth Estimation Approach I}

In order to detect a signal of interest using these embedded signatures, a receiving node can employ the low complexity single-cycle detector described in Section II-B. Upon detection of a signature in this way, OBW estimation can be achieved simply by examining the total number of features which comprise the signature. We will call this approach direct bandwidth estimation.

The use of BUs to realize bandwidth-adaptive waveforms is similar to the approach used in the IEEE 802.16e-2005 OFDMA-PHY specifications to achieve scalability [12]. Here, a fixed subcarrier spacing is employed to balance delay

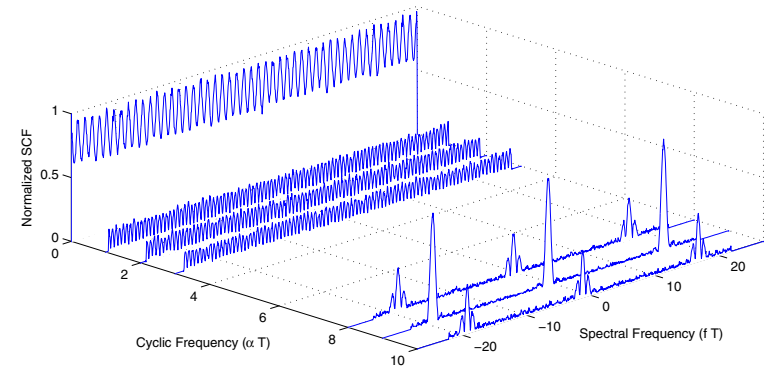

Fig. 4. Normalized SCF of OFDM waveform generated using 3 bandwidth units (BUs).

spread and Doppler spread requirements and the number of subcarriers scales with the channel bandwidth to be used. Table I illustrates the channel bandwidths and total number of subcarriers (FFT size) specified.

TABLE I

IEEE 802.16E-2005 SCALABLE OFDMA-PHY SPECIFICATIONS.

\begin{tabular}{|c|c|c|c|c|}
\hline Channel Bandwidth (MHz) & 1.25 & 5 & 10 & 20 \\
\hline FFT Size & 128 & 512 & 1024 & 2048 \\
\hline
\end{tabular}

In designing the $\mathrm{BU}$ to be used in an adaptive waveform, tradeoffs between bandwidth resolution, multipath robustness, system overhead and numbers of unique signatures must be considered. Given a fixed subcarrier spacing, choice of a large $\mathrm{BU}$ bandwidth will result in a greater number of subcarriers per BU. As the number of unique signatures which can be generated using $J$ subcarriers is equal to $J-2 M+1$ where $M$ is the number of mapped carriers, a larger BU bandwidth permits a greater number of unique signatures to be used. A large BU bandwidth will also decrease the bandwidth resolution which can be achieved using an adaptive waveform. This may limit the efficiency with which a DySPAN system can use white space spectrum. In terms of multipath fading, it has been shown that signatures with greater numbers of cyclostationary features provide greater robustness for signal detection [13]. For a given signal bandwidth, the choice of BU bandwidth will determine the number of features embedded. Thus, choice of a large BU bandwidth will reduce the number of unique features and may reduce detection robustness under multipath fading conditions. However, choice of a large BU bandwidth will also reduce the system overhead incurred as a result of using embedded signatures, a key consideration in the design of any wireless system.

\section{B. Bandwidth Estimation Approach II}

The direct bandwidth estimation approach is relatively straightforward and permits receiving nodes to determine the signal bandwidth without requiring timing or frequency synchronization. However, drawbacks of this approach include the limited bandwidth resolution which can be achieved and the possibility of a bandwidth estimation error in the event of a deep fade at just one of the embedded features. 
In order to overcome these drawbacks, an alternative approach to bandwidth estimation may be adopted. Each bandwidth unit used to generate a waveform effectively forms a subchannel with it's own embedded cyclostationary signature. It has been shown that these signatures may be used to achieve signal detection, identification and carrier frequency acquisition. Thus, upon detection of just one BU signature, a receiving node may choose to synchronize with that subchannel alone and demodulate the data carried therein. Within each BU subchannel, a short control header $(\mathrm{CH})$ can be used to carry information on the bandwidth and carrier frequency currently being used for the overall signal. In this way, upon demodulation of a single control header within one BU subchannel, a receiving node can determine the properties of the overall signal and establish a communications link. The use of control headers in this way is illustrated in Fig. 5.

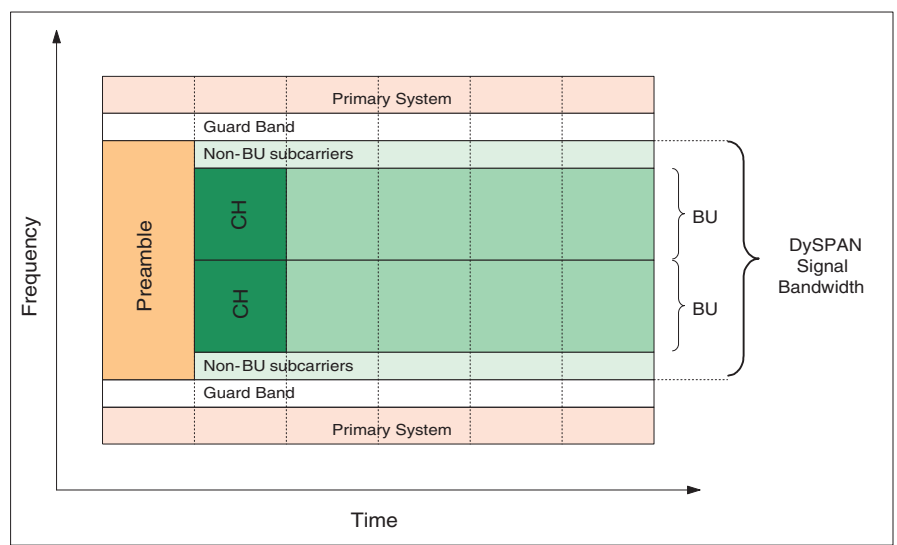

Fig. 5. Use of control headers within BU subchannels.

This use of subchannelization and control headers is similar to the approach proposed for the IEEE 802.22 standard for DySPAN operation in licensed TV bands [14]. In the context of IEEE 802.22, subchannel locations are fixed according to the TV channels being used and so the process of signal acquisition is greatly simplified. In the more general case however, cyclostationary signatures can be employed to support the establishment and maintenance of communication links using waveforms of arbitrary bandwidth and carrier frequency.

Direct bandwidth estimation relies upon detection of all cyclostationary features embedded in a signal of interest. In contrast, the use of control headers means that receiving nodes must detect just one feature in order to synchronize with a BU subchannel and derive the properties of the overall waveform. In addition, the use of control headers in this way overcomes the issue of limited bandwidth resolution associated with the direct approach. Using the direct approach, signals may only be generated as integer multiples of the BU bandwidth. Using control headers, signals may also be generated using one or more BUs however additional non-BU subcarriers can be employed to achieve arbitrary signal bandwidths. Fig. 5 illustrates the use of non-BU subcarriers in this way.

While there are a number of advantages associated with the use of control headers, there are also a number of drawbacks. Firstly, control headers impose an additional system overhead. Overhead can be reduced for a given signal bandwidth by reducing the number of BUs and increasing the numbers of non-BU carriers, however this approach may reduce signal detection performance under multipath fading conditions. Secondly, control headers must be successfully received and demodulated before the signal carrier frequency and bandwidth can be determined. This may require additional receiver complexity and may not be possible at low signal to noise ratio (SNR) levels.

\section{Simulations}

Monte Carlo simulations were carried out in order to examine bandwidth estimation performance using both the direct approach and the control header approach under multipath fading conditions. Parameters used for the simulation are presented in Table II.

TABLE II

SimUlation PARAMETERS

\begin{tabular}{|c|c|}
\hline Overall FFT Size & 1024 \\
\hline Subcarriers per BU & 64 \\
\hline Mapped subcarriers per BU & 3 \\
\hline Cyclic prefix & $1 / 8$ \\
\hline Observation time & 60 symbol durations \\
\hline Channel model & $\begin{array}{c}\text { Exponentially decayed 10-path } \\
\text { Rayleigh fading channel. } \\
\text { (Maximum Doppler freq. } f_{D} \approx 0 \mathrm{~Hz}\end{array}$ \\
\hline
\end{tabular}

Signals were generated using between 1 and 15 bandwidth units (BUs), each comprising 64 subcarriers. Within each BU a cyclostationary signature was embedded using a mapped set of 3 subcarriers. A mapping separation, $p=16$ was used for each signature. For each generated signal, the single-cycle signature detector (see eq. 13) was used to detect each embedded feature. An exponentially decayed 10-path Rayleigh fading channel model was used and decay factors, $\beta$, of between $-1 \mathrm{~dB}$ and $-3 \mathrm{~dB}$ were simulated. This channel model is illustrated in Fig. 6. White Gaussian noise was also added for $E_{s} / N_{0}$ of between -10 and $10 \mathrm{~dB}$.

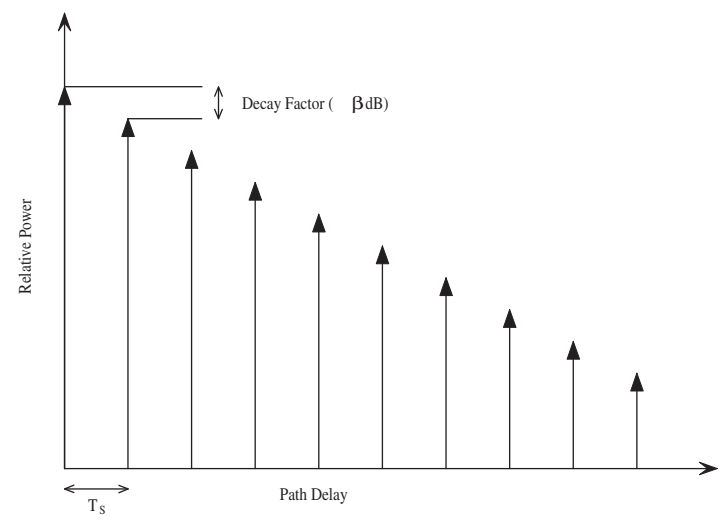

Fig. 6. Exponential decay channel model. 
1000 tests were carried out for each decay factor and value of $E_{s} / N_{0}$. A detection threshold of 0.25 was adopted and bandwidth estimation was carried out using both approaches.

For the direct estimation approach the signal bandwidth was estimated as

$$
B_{\text {est }}=\left(f_{\text {sigmax }}-f_{\text {sigmin }}\right)+B_{B U}
$$

where $f_{\text {sigmax }}$ is the spectral frequency of the highest detected feature, $f_{\text {sigmin }}$ is the spectral frequency of the lowest detected feature and $B_{B U}$ is the BU bandwidth. A probability of correct bandwidth estimation, $P_{b}$, was calculated over all tests. Results are illustrated in Fig. 7.

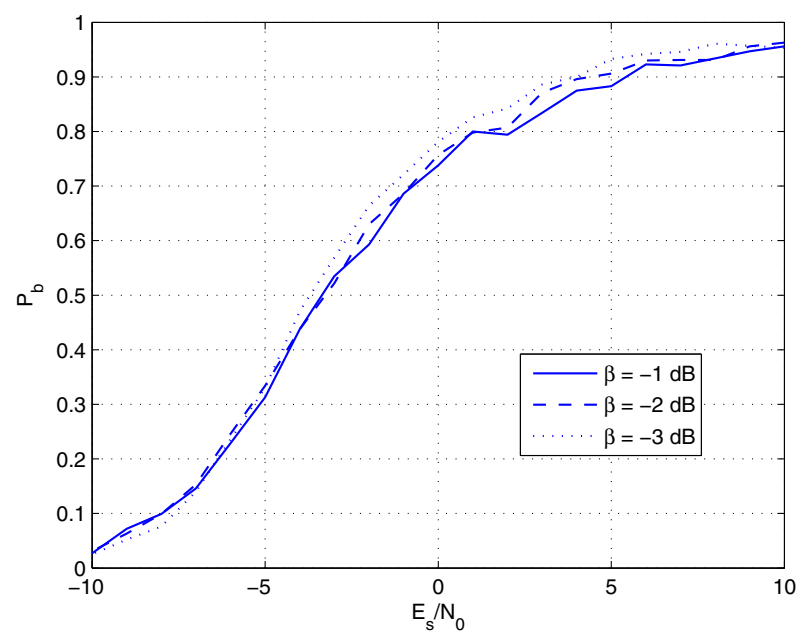

Fig. 7. Direct approach bandwidth estimation performance.

Results show that a $P_{b} \approx 95 \%$ can be achieved for $E_{s} / N_{0}=10 d B$ under multipath fading conditions using the direct bandwidth estimation approach. It can be seen that this performance deteriorates with decreasing $E_{s} / N_{0}$ with $P_{b} \approx 75-80 \%$ at $E_{s} / N_{0}=0 d B$. Below this level, performance falls sharply.

Estimation performance also decreases slightly with worsening multipath conditions, simulated by a smaller decay factor, $\beta$, in our channel model. At $E_{s} / N_{0}=5 d B$ and $\beta=-3 d B$, $P_{b} \approx 92 \%$. However for $\beta=-1 d B, P_{b}$ falls to $\approx 88 \%$.

For the control header approach, just one correct feature detection is required in order to locate a BU subchannel. Simulations were carried out as before, however in this case a probability of correct detection of just a single feature, $P_{d}$, was calculated over all tests. Results are illustrated in Fig. 8.

Results for single feature detection show that a probability of correct detection, $P_{d} \approx 100 \%$ can be achieved even at low levels of $E_{s} / N_{0}$ and under multipath fading. For $E_{s} / N_{0}<$ $-3 d B$ however, detection performance falls sharply.

Overall, simulation results show that bandwidth estimation for DySPAN systems can be achieved using embedded cyclostationary signatures under multipath fading conditions. Using the direct bandwidth estimation approach, it was seen

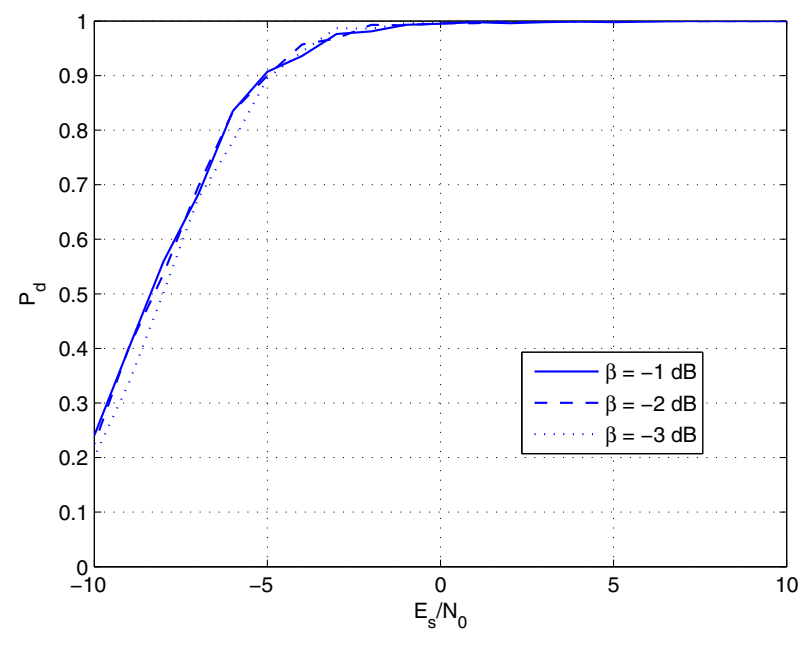

Fig. 8. Single feature detection performance for control header approach bandwidth estimation.

that robust performance can be achieved for $E_{s} / N_{0}>5 \mathrm{~dB}$. In contrast, the control header approach requires successful detection of just one embedded feature to locate a subchannel and so provides good performance for $E_{s} / N_{0}>-3 \mathrm{~dB}$. However, it should be noted that in order to determine the bandwidth of the overall signal using this approach, a receiving node must successfully demodulate a control header within that subchannel. At low levels of $E_{s} / N_{0}$ this may not be possible. Typically a receiving node will perform bandwidth estimation in order to establish a communication link. In the case where a control header cannot be successfully received, $E_{s} / N_{0}$ levels may be too low for that link to be established.

\section{CONCLUSIONS}

This paper has shown that intentionally embedded cyclostationary signatures provide a powerful tool for realising bandwidth-adaptive waveforms for dynamic spectrum access.

Using the concept of a bandwidth unit (BU), a novel approach for the generation of flexible-bandwidth multi-carrier waveforms was proposed. By embedding cyclostationary features within these BUs, it was shown that occupied bandwidth estimation at receiving DySPAN nodes can be achieved without the need for static control channels.

Two approaches were proposed for OBW estimation using embedded cyclostationary features. The first, direct, approach involves the detection of all embedded features and does not require synchronization with the signal of interest. The second approach exploits subchannelization of the signal of interest and uses control headers transmitted upon these subchannels to convey signal bandwidth and carrier frequency information. Using Monte Carlo simulation results, it was shown that both approaches may be successfully employed to achieve bandwidth estimation for adaptive multi-carrier waveforms. 


\section{ACKNOWLEDGEMENTS}

This material is based upon work supported by Science Foundation Ireland under Grant No. 03/CE3/I405 as part of the Centre for Telecommunications Value-Chain Research (CTVR) at Trinity College Dublin, Ireland.

\section{REFERENCES}

[1] T. A. Weiss and F. K. Jondral, "Spectrum pooling: an innovative strategy for the enhancement of spectrum efficiency," IEEE Communications Magazine, vol. 42, no. 3, pp. 8-14, Mar. 2004.

[2] B. Farhang-Boroujeny and R. Kempter, "Multicarrier communication techniques for spectrum sensing and communication in cognitive radios [cognitive radio communications]," Communications Magazine, IEEE, vol. 46, no. 4, pp. 80-85, 2008.

[3] D. Sarath, K. Nolan, P. Sutton, and L. Doyle, "Exploring the reconfigurability options of multi-carrier cdma in cognitive radio systems," in Personal, Indoor and Mobile Radio Communications, 2007. PIMRC 2007. IEEE 18th International Symposium on, 2007, pp. 1-5.

[4] K. Nolan, P. Sutton, L. Doyle, T. Rondeau, B. Le, and C. Bostian, "Demonstration and analyses of collaboration, coexistence, and interoperability of cognitive radio platforms," in Consumer Communications and Networking Conference, 2007. CCNC 2007. 4th IEEE, 2007, pp. 1149-1153.

[5] P. D. Sutton, K. E. Nolan, and L. E. Doyle, "Cyclostationary signatures for rendezvous in ofdm-based dynamic spectrum access networks," in New Frontiers in Dynamic Spectrum Access Networks, 2007. DySPAN 2007. 2nd IEEE International Symposium on, 17-20 April 2007, pp. $220-231$.

[6] P. Sutton, K. Nolan, and L. Doyle, "Cyclostationary signatures in practical cognitive radio applications," Selected Areas in Communications, IEEE Journal on, vol. 26, no. 1, pp. 13-24, 2008.

[7] W. Gardner, "Signal interception: a unifying theoretical framework for feature detection," IEEE Transactions on Communications, vol. 36(8), pp. 897-906, 1988.

[8] _ - Introduction to Random Processes with Applications to Signals and Systems, W. Gardner, Ed. MacMillan, 1985.

[9] K. Nolan, L. Doyle, P. Mackenzie, and D. O‘Mahony, "Fluid wireless dynamic spectrum allocation and spectrum-monitoring application using reconfigurable radio and OFDM," in Proceedings of the 2005 Software Defined Radio Forum (SDR Forum) Technical Conference, Anaheim, CA, USA, November 2005.

[10] D. Vučić, M. Obradović, and D. Obradović, "Spectral correlation of OFDM signals related to their PLC applications," in 6th International Symposium on Power-Line Communications and Its Applications, ISMPLC, March 27-29 2002.

[11] B. Sadler and A. Dandawate, "Nonparametric estimation of the cyclic cross spectrum," Information Theory, IEEE Transactions on, vol. 44, no. 1, pp. 351-358, Jan. 1998.

[12] "IEEE standard for local and metropolitan area networks Part 16: Air interface for fixed broadband wireless access systems, IEEE 802.162005," IEEE Standards Association, 2005.

[13] P. Sutton, J. Lotze, K. Nolan, and L. Doyle, "Cyclostationary signature detection in multipath rayleigh fading environments," in Cognitive Radio Oriented Wireless Networks and Communications, 2007. 2nd International Conference on, 2007.

[14] K. Challapali, C. Cordeiro, and D. Birru, "Evolution of spectrum-agile cognitive radios: first wireless internet standard and beyond," in WICON '06: Proceedings of the 2nd annual international workshop on Wireless internet. New York, NY, USA: ACM Press, 2006, p. 27. 